\title{
New look on the origin of cosmic rays
}

\author{
Ya.N. Istomin ${ }^{1,2, a}$ \\ 1 P.N. Lebedev Physical Institute, Leninsky Prospect 53, Moscow 119991, Russia \\ 2 Moscow Institute Physics and Technology, Institutskii per. 9, Dolgoprudnyi, Moscow region 141700, Russia
}

\begin{abstract}
From the analysis of the flux of high energy particles, $E>3 \cdot 10^{18} \mathrm{eV}$, it is shown that the distribution of the power density of extragalactic rays over energy is of the power law, $\bar{q}(E) \propto E^{-2.7}$, with the same index of 2.7 that has the distribution of Galactic cosmic rays before the so called 'knee', $E<3 \cdot 10^{15} \mathrm{eV}$. However, the average power of extragalactic sources, which is of $\mathcal{E} \simeq 10^{43} \mathrm{erg} \mathrm{s}^{-1}$, exceeds by at least two orders the power emitted by the Galaxy in cosmic rays, assuming that the density of galaxies is estimated as $N_{g} \simeq 1 \mathrm{Mpc}^{-3}$. Considering that such power can be provided by relativistic jets from active galactic nuclei with the power $\mathcal{E} \simeq 10^{45}-10^{46} \mathrm{erg} \mathrm{s}^{-1}$, we estimate the density of extragalactic sources of cosmic rays as $N_{g} \simeq 10^{-2}-10^{-3} \mathrm{Mpc}^{-3}$. Assuming the same nature of Galactic and extragalactic rays, we conclude that the Galactic rays were produced by a relativistic jet emitted from the Galactic center during the period of its activity in the past. The remnants of a bipolar jet are now observed in the form of bubbles of relativistic gas above and below the Galactic plane. The break, observed in the spectrum of Galactic rays ('knee'), is explained by fast escape of energetic particles, $E>3 \cdot 10^{15} \mathrm{eV}$, from the Galaxy because of the dependence of the coefficient of diffusion of cosmic rays on energy, $D \propto E^{0.7}$. The obtained index of the density distribution of particles over energy, $N(E) \propto E^{-2.7-0.7 / 2}=E^{-3.05}$, for $E>3 \cdot 10^{15} \mathrm{eV}$ agrees well with the observed one, $N(E) \propto E^{-3.1}$. The estimated time of the termination of the jet in the Galaxy is $4.2 \cdot 10^{4}$ years ago.
\end{abstract}

\section{Introduction}

The main hypothesis of the origin of cosmic rays in the Galaxy is the acceleration of charged particles to high energies on the fronts of shock waves formed by supernova explosions. To ensure the power of cosmic rays, observed in the Galaxy, which equals about $10^{41} \mathrm{erg} \mathrm{s}^{-1}$, it is necessary to transfer to accelerated particles about $15 \%$ of the kinetic energy of expanding shock waves. These strong shocks produce the universal spectrum of particle energy distribution, $N(E) \propto E^{-2}$. This allows to put together cosmic rays originating from different supernova, and forms the unique particle spectrum, extending from energies in a few units of $\mathrm{GeV}$ to energies $\simeq 10^{18} \mathrm{eV}$. The observed spectrum $N(E)$ significantly differs from the Universal spectrum produced via acceleration at shock fronts, that is explained by the propagation of cosmic rays in the Galaxy. It has the character of diffusion in space because of the scattering of charged particles by magnetic field inhomogeneities. The diffusion coefficient increases with particle energy, i.e. the lifetime of the fast particles in the Galaxy decreases. Therefore, the observed spectrum over energy differs from the spectrum given by sources, remaining a power law, $N(E) \propto E^{-\beta}$. But the value of $\beta$ is not constant, it changes from $\beta_{1}=2.7$ for $E<3 \cdot 10^{15} \mathrm{eV}$ to $\beta_{2}=3.1$ for $E>3 \cdot 10^{15} \mathrm{eV}$. The important circumstance is the fact that the spectrum at high energies becomes softer, not harder. This suggests that the source of cosmic rays at energies $E<3 \cdot 10^{15} \mathrm{eV}$ and at energies $E>3 \cdot 10^{15} \mathrm{eV}$ is single. It is possible that it is the superposition of two independent sources, if it would

a e-mail: istomin@lpi.ru be vice versa, $\beta_{2}<\beta_{1}$. But it is unlikely that the spectrum produced by one source at $E<3 \cdot 10^{15} \mathrm{eV}$ would cut off at higher energies, whereas another independent source at energies $E>3 \cdot 10^{15} \mathrm{eV}$ would cut off at lower energies, and they were joined at the same energy $E \simeq 3 \cdot 10^{15} \mathrm{eV}$.

The fact that the source of Galactic cosmic rays is single in the whole energy range, $E_{\min }<E<3 \cdot 10^{18} \mathrm{eV}$, suggests that if there are many in our Galaxy, they have the same universal properties. For the hypothesis of the origin of cosmic rays by supernova explosions this suggests that the shock wave is both strong enough and slightly loaded by cosmic rays. Otherwise, the spectral index of the accelerated particles will be different for different shock waves. This makes the hypothesis of the origin of cosmic rays by supernova explosions less attractive than previously assumed ([1,2]). In this article we do not discuss the paradigm of acceleration of Galactic cosmic rays by shock waves from supernova explosions. The last review made by Blasi [3] gives a good representation of this paradigm in many aspects. Observational data certainly show the acceleration of particles on the fronts of supernova shock waves. Is that enough to consider a hypothesis of the origin of Galactic cosmic rays by shock wave acceleration is proven? Here you can quote from Blasi's review: "Despite all this circumstantial evidence, no proof found yet that SNRs can accelerate CRs up to the knee energy."

If the source is single, it would also produce cosmic rays in other galaxies with characteristics similar to those observed in the Galaxy. The aim of our work is to analyze the properties of cosmic rays of superhigh energies $E>$ $3 \cdot 10^{18} \mathrm{eV}$ observed on the Earth, coming outside, from

(C) The Authors, published by EDP Sciences. This is an Open Access article distributed under the terms of the Creative Commons Attribution License 4.0 (http://creativecommons.org/licenses/by/4.0/). 
other galaxies, and compare them with the properties of Galactic cosmic rays.

\section{Ultrahigh energy cosmic rays}

The energy distribution of the flux of cosmic rays $I(E)$ observed on the Earth at energies $E>3 \cdot 10^{18} \mathrm{eV}$ noticeably deviates from the power law distribution, which is typical for lower energies, $3 \cdot 10^{15} \mathrm{eV}<E<$ $3 \cdot 10^{18} \mathrm{eV}$, where $I(E) \propto E^{-3.1}$. This region is called the 'ankle', here cosmic rays of Galactic origin are replaced by particles, the origin of which has extragalactic nature. They come from active galaxies located at large distances. In particular, there is identification of particles with energies $E \simeq 10^{19} \mathrm{eV}$ coming from the galaxy Cen A, located at a distance of $4.6 \mathrm{Mpc}$ (Auger Collaboration [4]), and correlation with active galactic nuclei $(\mathrm{AGN})$ within $\simeq$ $100 \mathrm{Mpc}$ (Auger Collaboration [5,6]). Charged particles (protons or nuclei) of such an energy, $E \simeq 10^{19} \mathrm{eV}$, have a large cyclotron radius, $r_{L}$, in the intergalactic magnetic field $B \simeq 10^{-9} \mathrm{G}$,

$$
r_{L}=\frac{E}{Z e B}=11 Z^{-1}\left(\frac{E}{10^{19} \mathrm{eV}}\right)\left(\frac{B}{1 n \mathrm{G}}\right)^{-1} \mathrm{Mpc} .
$$

The magnetic field changes its direction on the scale of the order of $1 \mathrm{Mpc}$, i.e. the correlation length of the magnetic field equals $l_{c} \simeq 1 \mathrm{Mpc}$. As a result, a charged particle moving to our Galaxy, walking distance, $r$, deviates from the initial direction by the angle $\Delta \theta$,

$$
\begin{aligned}
\Delta \theta= & \left(\frac{l_{c} r}{r_{L}^{2}}\right)^{1 / 2}=0.09 Z\left(\frac{E}{10^{19} \mathrm{eV}}\right)^{-1}\left(\frac{B}{1 n \mathrm{G}}\right) \\
& \times\left(\frac{l_{c}}{1 \mathrm{Mpc}}\right)^{1 / 2}\left(\frac{r}{1 \mathrm{Mpc}}\right)^{1 / 2} .
\end{aligned}
$$

Therefore, the propagation of cosmic rays of high energy from sources, that are closer than $r<100 \mathrm{Mpc}$, can be considered as rectilinear to a good approximation, while from more distant sources as diffusive. Let us find the distribution function, $N(t, \mathbf{r}, E)$, of cosmic rays of high energy, $E \simeq 10^{19} \mathrm{eV}$, over the space, $\mathbf{r}$, and the energy, $E$, considering that charged particles come to the given point from many sources in space located at points of $\mathbf{r}=\mathbf{r}_{i}$ and having powers $Q_{i}(E)$. Considering the distribution of sources in space as homogeneous on average, the particle distribution function of $N$ can be considered as isotropic, depending only on the distance, $r$. In addition, due to the large number of sources, the particle distribution can also be considered stationary, $N=N(r, E)$. We discuss two cases: rectilinear and diffusive propagation of particles.

\subsection{Rectilinear motion}

In this case, the equation for the distribution function of cosmic rays has the form

$$
-c \frac{\partial N}{\partial r}+\frac{\partial}{\partial E}\left(\frac{d E}{d t} N\right)=\sum_{i} \frac{Q_{i}(E)}{4 \pi r_{i}^{2}} \delta\left(r-r_{i}\right) .
$$

Here $c$ is the velocity of the light, $\delta(x)$ is the Dirac delta function. The value of $d E / d t$ is energy losses of particles. They consist of two parts: losses connected with the interaction of energetic particles with the relic radiation and losses associated with the expansion of the Universe. To the first, it is the so called GZK effect (Greisen [7]; Zatsepin \& Kuz'min [8]) - pion production in the reaction $p+\gamma \rightarrow N+\pi$. The characteristic particle energy of this process is $E_{\pi}=\mu c^{2}\left(1+\mu / m_{p}\right) m_{p} c^{2} / 2 T \simeq 4$. $10^{20} \mathrm{eV}\left(m_{p}\right.$ is the mass of the proton, $\mu$ is the mass of the muon, $T$ the temperature of the relic radiation). The interaction of a proton with a photon produces a neutral $\pi$-meson, the interaction of a photon with a nucleus can also produce charged $\pi$-mesons. At lower energies the generation of electron-positron pairs, $p+\gamma=p+e^{+}+$ $e^{-}$becomes significant. The characteristic particle energy of this reaction is $E_{e}=m_{e} c^{2} m_{p} c^{2} / T \simeq 2.1 \cdot 10^{18} \mathrm{eV}\left(m_{e}\right.$ is the electron mass). Energy losses in photo-pion reactions are well described by the expression (Stanev et al. [9])

$$
\left(\frac{d \epsilon}{d t^{\prime}}\right)_{\pi}=-(1+\epsilon) \exp \left(-\epsilon^{-1}\right)
$$

Here we introduced the dimensionless energy $\epsilon=$ $E / E_{\pi}=E / 4 \cdot 10^{20} \mathrm{eV}$ and the dimensionless time $t^{\prime}=$ $c t / L$, where $L$ is the characteristic distance, beyond which a particle loses the energy of the order of its initial value, $L=13.7 \mathrm{Mpc}$. These are convenient distances to measure in the same units, $r^{\prime}=r / L$. The energy losses due to the generation of electron-positron pairs (see the paper by Berezinsky, Gazizov \& Grigorieva [10]) can be approximated by the expression, which well describes that near the maximum of its relative losses,

$$
\begin{aligned}
\left(\frac{d \epsilon}{d t^{\prime}}\right)_{e} & =-a \epsilon\left(\epsilon_{e}^{s}+b \epsilon_{e}^{p}\right)^{-1} \exp \left(-\epsilon_{e}^{-1}\right), \\
\epsilon_{e} & =E / E_{e}=190 \epsilon .
\end{aligned}
$$

Here indices $s$ and $p$ are $s=-1.5, p=0.6$, and constants $a$ and $b$ are $a=4.5 \cdot 10^{-4}, b=8.4 \cdot 10^{-3}$ respectively. Adiabatic particle losses associated with the general expansion of the Universe are described by the Hubble law, $d E / d t=-H E$, where $H$ is the Hubble constant, $H=72 \mathrm{kms}^{-1} / \mathrm{Mpc}$. In dimensionless variables adiabatic losses are

$$
\left(\frac{d \epsilon}{d t^{\prime}}\right)_{a}=-\alpha \epsilon, \alpha=3.3 \cdot 10^{-3} .
$$

The total losses of particle energy are equal to

$$
\begin{aligned}
\frac{d \epsilon}{d t^{\prime}}= & -(1+\epsilon) \exp \left(-\epsilon^{-1}\right)-a \epsilon\left(\epsilon_{e}^{s}+b \epsilon_{e}^{p}\right)^{-1} \\
& \times \exp \left(-\epsilon_{e}^{-1}\right)-\alpha \epsilon .
\end{aligned}
$$

Although the value of $\alpha$ is small, the adiabatic energy losses become important for particles of not very high energies, $\epsilon_{e}<(\alpha / a)^{-1 / s} \simeq 3, E<6 \cdot 10^{18} \mathrm{eV}$, when interaction with relict photons becomes insignificant.

It should also be mentioned that in addition to relic photons in the intergalactic environment, there also exists the light from galaxies, which contributes to the energy losses of cosmic rays. But, as shown by calculations, their contribution is small compared with that of the relic radiation (Aloisio, Berezinsky \& Grigorieva [11]).

Let us introduce the dimensionless values of the power of the sources and the distribution function, 
$Q_{i}^{\prime}=Q_{i} L / c, N^{\prime}=N L^{3}$. The dimensionless variables (with index $/$, which we omit further) will appear in Eq. (1). We also introduce the variable $\tau$ instead of $\epsilon$,

$$
\tau=\int_{\epsilon}^{\epsilon^{\prime}} \frac{d x}{|d \epsilon / d t|_{\epsilon=x}} .
$$

The value of $\tau$ is the time during which a particle loses its energy from the initial value $\epsilon^{\prime}$ to the current one $\epsilon$. As a result, Eq. (1) will take the form

$$
\begin{gathered}
\frac{\partial}{\partial r}\left(\left|\frac{d \epsilon}{d t}\right| N\right)-\frac{\partial}{\partial \tau}\left(\left|\frac{d \epsilon}{d t}\right| N\right) \\
=-\sum_{i} \frac{Q_{i}(\epsilon)\left|\frac{d \epsilon}{d t}\right|}{4 \pi r_{i}^{2}} \delta\left(r-r_{i}\right) .
\end{gathered}
$$

Integrating Eq. (6) over $r$ from 0 to $r_{m}$ with the constant $\operatorname{sum} r+\tau=$ const, we get

$$
\begin{aligned}
N(r=0, \epsilon)= & \frac{1}{|d \epsilon / d t|} \sum_{i} \frac{Q_{i}\left(\epsilon_{i}\right)\left|\frac{d \epsilon}{d t}\right|_{\epsilon=\epsilon_{i}}}{4 \pi r_{i}^{2}} \\
& +\frac{|d \epsilon / d t|_{\epsilon=\epsilon_{m}}}{|d \epsilon / d t|} N\left(\epsilon_{m}, r_{m}\right) .
\end{aligned}
$$

The value of $r_{m}$ is the distance to which the approximation of rectilinear propagation is valid, $r_{m} \simeq$ $100 \mathrm{Mpc} / 13.7 \mathrm{Mpc}=7.3$. The energy $\epsilon_{m}$ is determined by the ratio

$$
r_{m}=\int_{\epsilon}^{\epsilon_{m}} \frac{d x}{|d \epsilon / d t|_{\epsilon=x}} .
$$

In Eq. (7) the first term is the contribution of the sources of cosmic rays of superhigh energies, located at distances $r<r_{m}$ from the Galaxy, whose particles are observed in the Galaxy $(r=0)$. Because, according to the analysis by Takami \& Sato [12], the density of such sources is of $10^{-2}-10^{-4} \mathrm{Mpc}^{-3}$, and the number of these sources in the sphere of radius $r_{m}$ is significantly greater than unity, it is possible to go from the summation to the integration over volume, $4 \pi \int_{0}^{r_{m}} r_{i}^{2} d r_{i}$, introducing the average density of sources $\bar{q}(\epsilon)$. The value of energy, on which the quantity $\bar{q}(\epsilon)$ depends, is the initial energy of a particle, which overcomes the distance $r_{i}$ from the source to the Galaxy, $\epsilon=\epsilon_{i}$,

$$
r_{i}=\int_{\epsilon}^{\epsilon_{i}} \frac{d x}{|d \epsilon / d t|_{\epsilon=x}}
$$

However, at constant energy $\epsilon$ the distance $r_{i}$ and the energy $\epsilon_{i}$ are connected, so the integration over $r_{i}$ can be replaced by the integration over $\epsilon_{i}$. The result is

$$
\frac{1}{|d \epsilon / d t|} \sum_{i} \frac{Q_{i}\left(\epsilon_{i}\right)\left|\frac{d \epsilon}{d t}\right|_{\epsilon=\epsilon_{i}}}{4 \pi r_{i}^{2}}=\frac{\int_{\epsilon}^{\epsilon_{m}} \bar{q}\left(\epsilon^{\prime}\right) d \epsilon^{\prime}}{|d \epsilon / d t|} .
$$

Thus, the distribution of cosmic rays of high energy, $E>$ $3 \cdot 10^{18} \mathrm{eV}$, observed in the Galaxy, is largely determined by the loss function $|d \epsilon / d t|$ (Eq. (5)) in the intergalactic space, but not by the sources, the distribution of which over the energy $\bar{q}(\epsilon)$ is in average form.

\subsection{Diffusive motion}

In order to determine the contribution of distant $\left(r>r_{m}\right)$ sources into the distribution of cosmic rays observed in our Galaxy (the second term in Eq. (7)) we have to consider the diffusion region $r>r_{m}$. The equation for the distribution function $N(r, \epsilon)$ is

$$
\frac{\partial}{\partial E}\left(\frac{d E}{d t} N\right)-\frac{D}{r^{2}} \frac{\partial}{\partial r}\left(r^{2} \frac{\partial N}{\partial r}\right)=\sum_{i} \frac{Q_{i}(E)}{4 \pi r_{i}^{2}} \delta\left(r-r_{i}\right) .
$$

Here $D$ is the coefficient of diffusion of cosmic rays in intergalactic space. As well as in the Galaxy, it can depend on the particle energy. Let us suppose that this dependence is a power law with some index $\kappa, D=D_{0} E^{\kappa}$. Also as before, we introduce the dimensionless variables: the distances are measured in $L$, time - in $L / c$, the energy in $4 \cdot 10^{20} \mathrm{eV}$, the diffusion coefficient - in units of $D_{0}^{\prime}=$ $D_{0}\left(4 \cdot 10^{20} \mathrm{eV}\right)^{\kappa} / L c$. We introduce also the effective time $\tau_{D}$,

$$
\tau_{D}=\int_{\epsilon}^{\epsilon^{\prime}} \frac{x^{\kappa} d x}{|d \epsilon / d t|_{\epsilon=x}} .
$$

As a result, Eq. (8) becomes

$$
\begin{gathered}
\frac{\partial}{\partial \tau_{D}}\left(\left|\frac{d \epsilon}{d t}\right| N\right)-\frac{D_{0}}{r^{2}} \frac{\partial}{\partial r}\left(r^{2} \frac{\partial}{\partial r}\left(\left|\frac{d \epsilon}{d t}\right| N\right)\right) \\
=\epsilon^{-\kappa}\left|\frac{d \epsilon}{d t}\right| \sum_{i} \frac{Q_{i}(\epsilon)}{4 \pi r_{i}^{2}} \delta\left(r-r_{i}\right) .
\end{gathered}
$$

Knowing the Green function of the equation of diffusion in a spherical region $0<r<\infty$ (the diffusion approximation includes also the region $0<r<r_{m}$ of the scale of the free path length),

$$
\begin{aligned}
G\left(r, r^{\prime}, t\right)= & \frac{1}{\left(4 \pi D_{0} t\right)^{1 / 2}} \frac{r^{\prime}}{r}\left\{\exp \left[-\frac{\left(r-r^{\prime}\right)^{2}}{4 D_{0} t}\right]\right. \\
& \left.-\exp \left[-\frac{\left(r+r^{\prime}\right)^{2}}{4 D_{0} t}\right]\right\} .
\end{aligned}
$$

Integrating this with the right hand part of equation (9) over $r^{\prime}$ and $\tau_{D}$, then, as before, going from the summation over $i$ to the integration over $r_{i}$ from $r_{m}$ to infinity, introducing again the density of sources $\bar{q}(\epsilon)$, and transforming the integration over $\tau_{D}$ to the integration over $\epsilon^{\prime}$, we get

$$
N\left(r_{m}, \epsilon\right)=\frac{1}{|d \epsilon / d t|} \int_{\epsilon}^{\epsilon_{0}} \bar{q}\left(\epsilon^{\prime}\right) \Phi\left[\left(\frac{r_{m}^{2}}{4 D_{0} \tau_{D}\left(\epsilon^{\prime}\right)}\right)^{1 / 2}\right] d \epsilon^{\prime} .
$$

Here the function $\Phi(y)$ is equal to

$$
\begin{aligned}
\Phi(y)= & 2-\frac{2}{\pi^{1 / 2}} \int_{0}^{2 y} \exp \left(-z^{2}\right) d z \\
& +\frac{1}{\pi^{1 / 2} y}\left[1-\exp \left(-4 y^{2}\right)\right] .
\end{aligned}
$$

It varies monotonically from the value $\Phi=2$ at $y=0$ to $\Phi=1$ for $y>>1$. And the fast transition of $\Phi$ to the value $\Phi \simeq 1$ occurs already at $y \simeq 2$. The typical value of $y$ is the ratio of the length of the mean free path length of particles $r_{m}$ to the path $L$, travelling along which a particle 
loses a significant part of its energy, $y \simeq r_{m} / L=7.3$. In addition, the large time $\tau_{D}$, small $y$, corresponds to high initial energies of particle $\epsilon^{\prime}$. Because the source power $\bar{q}(\epsilon)$ decreases with energy, and, as we will see, rather fast, $\bar{q}(\epsilon) \propto \epsilon^{-2.7}$, then the contribution of the function $\Phi(y)$ at small values of its argument into the integral (Eq. (11)) is small. Thus, we can consider $\Phi \simeq 1$ in the expression (Eq. (11)). Altogether, the required distribution function of particles on the boundary of $r=r_{m}$ at $\epsilon=\epsilon_{m}$ equals

$$
N\left(r_{m}, \epsilon_{m}\right)=\frac{1}{|d \epsilon / d t|_{\epsilon=\epsilon_{m}}} \int_{\epsilon_{m}}^{\epsilon_{0}} \bar{q}\left(\epsilon^{\prime}\right) d \epsilon^{\prime}
$$

The value of $\epsilon_{0}$ is the maximum energy of particles in sources.

\section{Energy distribution of extragalactic particles observed in the Galaxy}

Substituting the resulting distribution (Eq. (12)) into the expression (Eq. (7)), we obtain the sought distribution function of extragalactic cosmic rays, observed in the Galaxy,

$$
N(r=0, \epsilon)=\frac{\int_{\epsilon}^{\epsilon_{0}} \bar{q}\left(\epsilon^{\prime}\right) d \epsilon^{\prime}}{|d \epsilon / d t|} .
$$

Equation (13) has a simple physical meaning: the particle flux in the energy space, $N|d \epsilon / d t|$, is equal to the total flux produced by sources $Q_{i}$. Moreover, since we have many sources, their fluxes are summing, ranging from close sources, providing a full range of energies from $\epsilon=\epsilon_{\min }$ up to the maximum one $\epsilon=\epsilon_{0}$, to distant sources from which we observe only large initial energy $\epsilon \simeq$ $\epsilon_{0}$. Suppose that the distribution $\bar{q}(\epsilon)$ is the power law function, $\bar{q}(\epsilon)=q_{0} \epsilon^{-\beta}$. Then the distribution function $N(\epsilon)$ is equal to

$$
\begin{aligned}
N(\epsilon)= & \frac{q_{0}}{\beta-1} \frac{\epsilon^{-\beta+1}-\epsilon_{0}^{-\beta+1}}{F(\epsilon)}, \\
F(\epsilon)= & (1+\epsilon) \exp (-1 / \epsilon) \\
& +a \epsilon\left(\epsilon_{e}^{s}+b \epsilon_{e}^{p}\right)^{-1} \exp \left(-1 / \epsilon_{e}\right)+\alpha \epsilon .
\end{aligned}
$$

The graph of the function $\epsilon^{3} N(\epsilon)$ for $\epsilon_{0}=25, E_{0}=$ $10^{22} \mathrm{eV}$, and $\beta=2.7$ is shown on Fig. 1. We also show here the experimental data (points) obtained by Telescope Array (see the Fig. 2). One can see that at energies $E<10^{18} \mathrm{eV}$ and $E>4 \cdot 10^{20} \mathrm{eV}$ the distribution $N(\epsilon)$ is the power law, $N(\epsilon) \propto \epsilon^{-\beta}$. The same slope is observed at the intermediate energies, in the region of the maximum relative losses of energy, $(d E / d t) / E$, on the birth of electron-positron pairs, $E \simeq 10^{19} \mathrm{eV}$. In the region $0.2<\epsilon<1, N$ grows exponentially with decreasing energy, $N(\epsilon)=q_{0} \epsilon^{-\beta+1} \exp (1 / \epsilon) /(\beta-1)$. According to observations summarized by Berezinsky [13], the flux of particles $I(\epsilon)=c N(\epsilon) / 4 \pi$ in the energy range $6 \cdot 10^{18} \mathrm{eV}<E<6 \cdot 10^{19} \mathrm{eV}, 1.5 \cdot 10^{-2}<$ $\epsilon<0.15$, is indeed the power law function of the energy with the index $\beta=2.7$. As an illustration of this we show here observations of the Telescope Array (Tinyakov [14]). One can see in Fig. 2 that the slope is indeed equal to $0.33 \pm 0.03$ in the range $6 \cdot 10^{18} e V<E<6 \cdot 10^{19} \mathrm{eV}$.

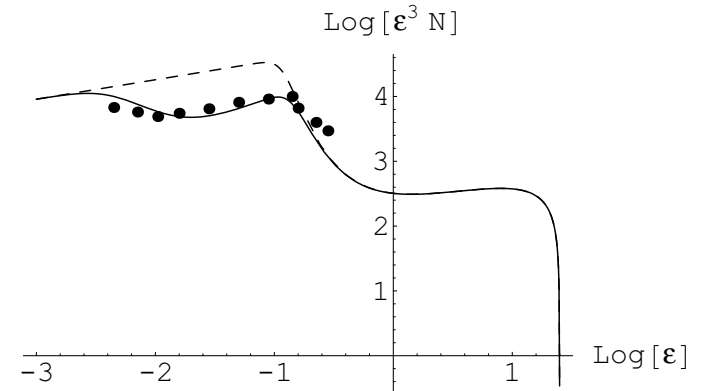

Figure 1. The function $\epsilon^{3} N(\epsilon), N(\epsilon)$ is given by the formula (14). Here we choose the value of maximum energy $E_{0}=$ $10^{22} \mathrm{eV}, \epsilon_{0}=25$, and the index $\beta=2.7$. The dashed line represents this function when electron-positron pair production is absent. Points are observational data by the Telescope Array (see Fig. 2).

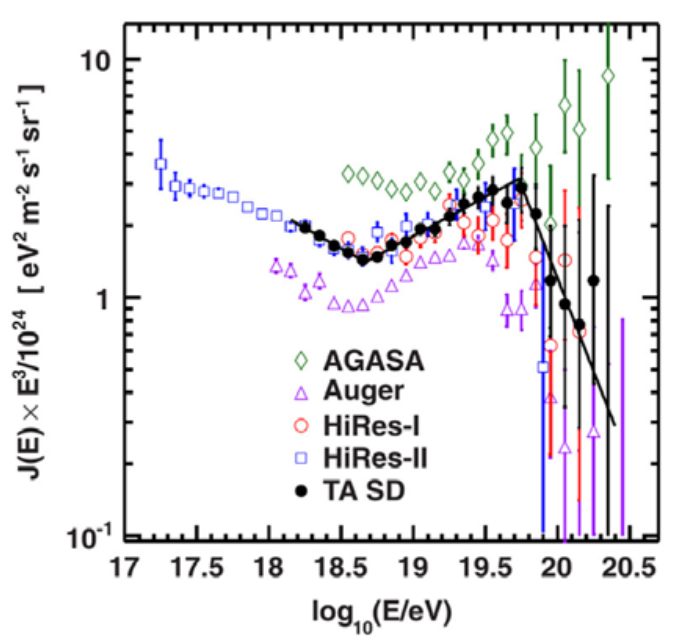

Figure 2. The spectrum of high energy cosmic rays observed by Telescope Array (black points) (Tinyakov [14]). In the range $6 \cdot 10^{18} \mathrm{eV}<E<6 \cdot 10^{19} \mathrm{eV}$ the spectrum is the power law function of the energy with the index $\beta=2.67 \pm 0.03$. Another points present also observations by different instruments.

When analyzing the data of observations it is convenient to use the function $\epsilon^{3} N(\epsilon)$, which clearly describes the transition from the Galactic distribution $\left(E^{3} I(E) \propto E^{-0.1}\right)$ for $E<3 \cdot 10^{18} \mathrm{eV}$ to the extragalactic one $\left(E^{3} I(E) \propto E^{0.3}\right)$ for $6 \cdot 10^{18} \mathrm{eV}<E<6 \cdot 10^{19} \mathrm{eV}$. The function $\epsilon^{3} N(\epsilon)$ has a maximum at $\epsilon=\epsilon_{1}=$ $0.1, E_{1}=4 \cdot 10^{19} \mathrm{eV}$ (see Fig. 2). At energies $\epsilon>\epsilon_{1}$ the function $\epsilon^{3} N(\epsilon)$ exponentially decreases not to zero, but to the minimum value at $\epsilon_{2}, \epsilon_{2} \simeq 1, E_{2} \simeq 4 \cdot 10^{20} \mathrm{eV}$. After that the distribution $N(\epsilon)$ continues to fall down by a power law manner with the same index $\beta, N(\epsilon) \propto \epsilon^{-\beta}$. The fall down of the function $\epsilon^{3} N(\epsilon)$ at energy $\epsilon_{1}$ to its value at $\epsilon_{2}$ is approximately one and half orders. The strong growth of the density of particles for $\epsilon<\epsilon_{2}$ is explained by the sharp decrease of the rate of energy losses of particles when braking by relict photons becomes small. Here particles are accumulated. The distribution of $N(E)$ for energies $E>4 \cdot 10^{20} \mathrm{eV}$ does not go exponentially to small values that can seem as the result of the GZK effect. Here we observe that the distribution of $N(E)$ repeats the distribution of extragalactic sources $\bar{q}(E)$, unless the maximum energy $E_{0}$ is not close to the energy $4 \cdot 10^{20} \mathrm{eV}$. However, at energies $E>4 \cdot 10^{20} \mathrm{eV}$ the observational 
data have great uncertainty, which does not allow to make a conclusion about the growth of the function $E^{3} N(E)$ at high energies $E>4 \cdot 10^{20} \mathrm{eV}$ under the condition $E_{0}>4 \cdot 10^{20} \mathrm{eV}$. The energy range $E<6 \cdot 10^{18} \mathrm{eV}$ (see Fig. 1), where the distribution $N(E)$ also reproduces the distribution of extragalactic sources $\bar{q}(E)$, is hidden by Galactic cosmic rays.

It should be noted that the solution of the kinetic equation in the form (13) is not only valid for protons, but also for arbitrary nuclei with atomic number $A, N=N_{A}$. One only needs to take into account the fact that sources of the nucleus $A$ are not only point sources $q^{s}$, in which nuclei are accelerated, but also the intergalactic space, in which photo-disintegration of nuclei occurs when they collide with photons,

$$
\begin{aligned}
A^{\prime}+\gamma= & A+N, \quad A^{\prime}>A \\
q_{A}(E)= & q_{A}^{s}-N_{A}(E) \sum_{A^{\prime}<A} v_{A, A^{\prime}}(E) \\
& +\sum_{A^{\prime}>A} N_{A^{\prime}}\left(m_{A^{\prime}} E / m_{A}\right) v_{A, A^{\prime}}\left(m_{A^{\prime}} E / m_{A}\right) .
\end{aligned}
$$

Here $v_{A, A^{\prime}}$ is the frequency of the disintegration of the nucleus $A^{\prime}$ into the nucleus $A$,

$$
v_{A, A^{\prime}}(E)=c \int_{E_{t h}}^{\infty} n_{p h} \sigma_{A, A^{\prime}}\left(E, E_{p h}\right) d E_{p h} .
$$

The value of $n_{p h}$ is the spectral density of photons in intergalactic space, $\sigma_{A, A^{\prime}}$ is the cross-section of photodisintegration, $E_{t h}$ is the threshold energy of a photon. In the expression (13) for the energy loss $d E / d t$ we should also add the energy loss of the nucleus during photo-disintegration, which is maximum at the energy of the nucleus $E_{A} / A \simeq E_{b} m_{p} c^{2} / 2 T \simeq 10^{19} \mathrm{eV}\left(E_{b}\right.$ is the binding energy of the nucleon $\mathrm{N}$ in the nucleus $A, E_{b} \simeq$ $10 \mathrm{MeV})$. Thus, the distribution of nuclei over energy is determined by the system of equations

$$
\begin{aligned}
\left|\frac{d E}{d t}\right|_{A} N_{A} & =\int_{E}^{E_{0}} q_{A}^{s}\left(E^{\prime}\right) d E^{\prime}-\int_{E}^{E_{0}} N_{A}\left(E^{\prime}\right) v^{t}\left(E^{\prime}\right) d E^{\prime} \\
& +\sum_{A^{\prime}>A} \int_{E}^{E_{0}} v\left(m_{A}^{\prime} E^{\prime} / m_{A}\right) N_{A^{\prime}}\left(m_{A}^{\prime} E^{\prime} / m_{A}\right) d E^{\prime}
\end{aligned}
$$

The value of $v^{t}(E)$ is a total frequency of destruction of the nucleus $A$ with formation of all nuclei $A^{\prime}<A, v^{t}(E)=$ $\sum_{A^{\prime}<A} v_{A, A^{\prime}}(E)$. The system of Eq. (15) can be simply solved for two limiting cases:

a) In sources at $E>10^{18} \mathrm{eV}$ protons are the main component. Then for protons the second and the third terms in the right hand side of (15) are equal to zero. The solution for $N_{p}(E)$ looks as described above, Eq. (14).

b) In sources at $E>10^{18} \mathrm{eV}$ iron is the main component. Then for $N_{F e}(E)$ we get

$$
\begin{aligned}
& \left|\frac{d E}{d t}\right|_{F e} N_{F e}(E)+\int_{E}^{E_{0}} v^{t}\left(E^{\prime}\right) N_{F e}\left(E^{\prime}\right) d E^{\prime} \\
& =\int_{E}^{E_{0}} q_{F e}^{s}\left(E^{\prime}\right) d E^{\prime} .
\end{aligned}
$$

The solution of (16) is

$N_{F e}\left|\frac{d E}{d t}\right|_{F e}=\int_{E}^{E_{0}} q^{s}\left(E^{\prime}\right) \exp \left\{-\int_{E}^{E^{\prime}} \frac{v^{t}\left(E^{\prime \prime}\right)}{|d E / d t|^{\prime \prime}} d E^{\prime \prime}\right\} d E^{\prime}$.

It is seen that the factor $\exp \left\{-\int_{E}^{E^{\prime}}\left(v^{t}\left(E^{\prime \prime}\right) /|d E / d t| "\right) d E^{\prime \prime}\right\}$ suppresses sources at high energies due to the destruction of iron in interstellar space into light nuclei. Near the maximum of relative losses, where $|d E / d t| \propto E$, the index $\beta$ in sources $\left(q^{s} \propto E^{-\beta}\right)$ must be less than the observed index $\beta^{o b s}, \beta<\beta^{o b s}=2.7$. Generally speaking, the factor $\exp \left\{-\int_{E}^{E^{\prime}}\left(v^{t}(E ") /|d E / d t| "\right) d E "\right\}$ is not an exponential function of the energy $E^{\prime}$, and if sources (galaxies) produce a power-law spectrum, the observed distribution of $N_{F e}(E)$ is not a power-law function. However, the average value of $\beta$, obtained by Monte Carlo simulation by Taylor, Ahlers \& Aharonian [15], is of the value of $\beta \simeq 1.8$. If we consider the effect of propagation of nuclei in the host galaxy, this suggests that in the accelerating machine the index $\beta \simeq 1$. Accelerating mechanisms, in which the main energy is released at maximum energies $E \simeq E_{0}$, are difficult to find in nature. As for other nuclei, the same suppressing factor $\exp \left\{-\int_{E}^{E^{\prime}}\left(v^{t}\left(E^{\prime \prime}\right) /|d E / d t| "\right) d E "\right\}$ associated with their disintegration, but to a less degree than for iron, will lead to an effective value of $\beta$ less than $\beta^{o b s}, 2.7<\beta<1.8$. This index will be different for different nuclei $A<A_{F e}$.

The statement that in the energy range where $|d E / d t| \propto E$ the observed spectrum of extragalactic cosmic rays $N(E)$ repeats the source spectrum $q(E) \propto$ $E^{-\beta}$, see (13), generally speaking, is true if the maximum energy $E_{0}$ for sources is larger, on average, than that in the range considered $6 \cdot 10^{18} \mathrm{eV}<E<6 \cdot 10^{19} \mathrm{eV}$. However, if $E_{0}$ is in this range, and is different for different sources, the index $\beta$ can differ from the observed value of $\beta^{\text {obs }}$. In order to find a connection of $\beta$ with $\beta^{\text {obs }}$ it is necessary to average Eq. (13) over different realizations of $E_{0}$.

$$
N(E) \propto\left\langle E^{-1}\left(E^{-\beta+1}-E_{0}^{-\beta+1}\right)\right\rangle .
$$

In this expression the contribution only gives sources that have $E_{0}$ larger than $E, E_{0}>E$. We introduce the probability $P\left(E_{0}\right)$ to have a given value of $E_{0}$,

$$
\langle\ldots\rangle=\int_{E}^{\infty} \ldots P\left(E_{0}\right) d E_{0}, \int_{E^{*}}^{\infty} P\left(E_{0}\right) d E_{0}=1 .
$$

For the distribution $N(E)$ to be an exponential function of $E, P\left(E_{0}\right)$ has also to be a power-law function $P\left(E_{0}\right)=$ $(r-1)\left(E^{*} / E_{0}\right)^{r} / E^{*}$. The index $r$ is not only larger than unit, but larger than two, $r>2$, in order for the average value of $E_{0}$ to be a finite value. The result is

$$
\left\langle E^{-1}\left(E^{-\beta+1}-E_{0}^{-\beta+1}\right)\right\rangle=\frac{\beta-1}{\beta+r-2} E^{-\beta-r+1} E^{* r-1} .
$$

Thus, $\beta=\beta^{o b s}-r+1<\beta^{o b s}-1<1.7$. To explain the observed power-spectrum of extragalactic cosmic rays at $E>6 \cdot 10^{18} \mathrm{eV}$ as a result of the superposition of different sources with different values of maximum energy $E_{0}$, we must have hard sources with $\beta<1.7$, that is probably small.

Let us now estimate the average power density of sources $\bar{q}$. Observations give the value of the extragalactic 
particle flux, where there is a maximum value of the product $E^{3} I(E)$ at the energy $E_{1}=4 \cdot 10^{19} \mathrm{eV}$,

$$
I\left(E_{1}\right)=4.7 \cdot 10^{-39} \mathrm{~cm}^{-2} \mathrm{~s}^{-1} \mathrm{sr}^{-1} \mathrm{eV}^{-1} .
$$

Accordingly, the density of particles $N(E), N(E)=$ $4 \pi I(E) / c$, is

$$
N\left(E_{1}\right)=5.9 \cdot 10^{25} \mathrm{Mpc}^{-3} \mathrm{eV}^{-1} .
$$

On the other hand, the dimensionless density of particles $N\left(\epsilon_{1}\right)$ and average power of sources $\bar{q}\left(\epsilon_{1}\right)$ are connected by the relation (14),

$$
\begin{aligned}
\bar{q}\left(\epsilon_{1}\right)= & (\beta-1)\left[\left(\epsilon_{1}^{-1}+1\right) \exp \left(-1 / \epsilon_{1}\right)+a\left(\epsilon_{e 1}^{-2}+b \epsilon_{e 1}^{0.6}\right)^{-1}\right. \\
& \left.\exp \left(-1 / \epsilon_{e 1}\right)+\alpha\right] N\left(\epsilon_{1}\right)=2 \cdot 10^{-2} N\left(\epsilon_{1}\right) .
\end{aligned}
$$

Bearing in mind that the dimensional quantity $\bar{q}$ is $c / L=$ $7.1 \cdot 10^{-16} s^{-1}$ times less than the dimensionless one, we get

$$
\bar{q}\left(E_{1}\right)=8.4 \cdot 10^{8} \mathrm{eV}^{-1} \mathrm{~s}^{-1} \mathrm{Mpc}^{-3} .
$$

Considering that, as well as in our Galaxy, the power law spectrum with index $\beta=2.7$ continues up to small energies $E_{\min } \simeq 5 \mathrm{GeV}$, we get

$$
\bar{q}(E)=8.4 \cdot 10^{8}\left(\frac{E}{4 \cdot 10^{19} \mathrm{eV}}\right)^{-2.7} \mathrm{eV}^{-1} \mathrm{~s}^{-1} \mathrm{Mpc}^{-3} .
$$

The total average power density of extragalactic sources $\mathcal{E}=\int_{E_{\min }}^{E_{0}} \bar{q}(E) E d E$ is equal to

$$
\mathcal{E}=1.8 \cdot 10^{43}\left(\frac{E_{\min }}{5 \mathrm{GeV}}\right)^{-0.7} \operatorname{erg~s}^{-1} \mathrm{Mpc}^{-3} .
$$

This value divided by the density of galaxies, $N_{g} \simeq$ $1 \mathrm{Mpc}^{-3}$, exceeds the power of the Galaxy in cosmic rays, $10^{41} \mathrm{erg} \mathrm{s}^{-1}$, by at least two orders of magnitude. If we assume that the sources of ultrahigh energy particles are active galactic nuclei with relativistic jets generated inside, the power of which is $\left(10^{45}-10^{46}\right) \mathrm{erg} \mathrm{s}^{-1}$ (Mao- $\mathrm{Li}$, et al. [16]), then their density in the Universe is of $N_{g} \simeq$ $\left(10^{-2}-10^{-3}\right) \mathrm{Mpc}^{-3}$. The same estimation of the density of extragalactic sources of cosmic rays follows also from the conditions of arrival isotropy of particles in the range of energies $E \simeq 10^{19} \mathrm{eV}$ (Abbasi et al. [17]).

\section{Galactic cosmic rays}

The most surprising fact, following from the previous consideration, is that the spectrum of the power density of extragalactic cosmic rays (18) has the the same slope as the Galactic cosmic ray density before the 'knee', $E<$ $3 \cdot 10^{15} \mathrm{eV}, \beta=2.7$. Let us note here that understanding the cosmic ray power density $\bar{q}(E)$ as the average power radiated by a galaxy as a whole, is proportional to the density of cosmic rays in a galaxy $N(E), \bar{q}(E) \propto N(E) S c$, where the value of $S$ is the galactic surface radiated cosmic rays. This indicates that the nature of the origin of cosmic rays in the Galaxy and in active galactic nuclei is the same. The formation of the spectrum with index $\beta=2.7$ in the Galaxy is explained by the fact that the index of the power law energy spectrum of source is of universal value $\beta=2$. This is valid as for the acceleration of particles on fronts of strong shock waves $([1,2])$, as well as for the acceleration at the base of the jets emitted near massive black holes in centers of galaxies (Istomin [18]).

Next, accelerated particles, spreading over a galaxy, are scattered by inhomogeneities of a magnetic field. Their motion becomes diffusive. Moreover, the coefficient of diffusion is larger for particles with larger energies, $D \propto$ $E^{\delta}, \delta \simeq 0.6$ (Ptuskin [19]). It should be noted that the index $\delta$ is the same for protons and for different nuclei. This follows from the general selfsimilarity considerations. The diffusion of charged particles by fluctuations of the magnetic field depends, in the general case, on three dimensional quantities: a particle velocity $v$, its Larmor radius $r_{L}$ and the correlation length of the random field $l_{c}$. Thus, $D=c l_{c} f\left(r_{L} / l_{c}\right)$, where $c$ (the speed of the light) is the speed of motion of a relativistic particle, $v=c$, $f$ is a dimensionless function of the ratio of a particle Larmor radius, $r_{L}=E / e Z B$, to the correlation length $l_{c}$. This function depends on the spectrum and amplitude of the random magnetic field. Because the various charged particles are scattered by the same magnetic field, the power-law index $\delta$ is the same for different nuclei. Only the diffusion coefficients can slightly vary at the same energy $E$. But the diffusion is the same for particles of the same rigidity $\mathcal{R}$ (Blasi \& Amato [20]), $r_{L}=\mathcal{R} / B$. The observed spectrum of Galactic cosmic rays under the assumption about the universality of the source gives the value of $\delta$ of 0.7. We are taking this value as following from the observations, regardless of other values obtained from theoretical and numerical calculations of the scattering of charged particles and of the paradigm of particle acceleration by shock waves from supernova explosions.

The density of particles is equal to the product of the power of a source, $Q$, to the lifetime of particles $\tau$, $N=Q \tau$. The lifetime is the escape time of particles from a galaxy, $\tau=R^{2} / D, R$ is the radius of a galaxy. Thus, $N(E) \propto E^{-2-0.7}$. There arises the question: why in our Galaxy does the spectral index of cosmic rays at energies $E>3 \cdot 10^{15} \mathrm{eV}$ deviate from the value of $\beta=2.7$ ? The spectrum becomes softer, $\beta=3$.1. If the source of cosmic rays in a galaxy is through strong shock waves from supernova explosions, it is not clear why the spectrum of high energy particles from active galaxies remains with index $\beta=2.7$ up to energies $\sim 10^{20} \mathrm{eV}$. It is more natural to assume that the source of cosmic rays is a jet emitted from an active galactic nucleus and whose power significantly exceeds the power transformed to particles by supernova explosions. Istomin [18] suggested that Galactic cosmic rays were produced by the jet, emitted from the center of the Galaxy. Giant bubbles of relativistic gas, observed above and below the Galactic plane, are remnants of this bipolar jet that existed previously. From the size of the bubbles it follows that the jet switched on 2.4. $10^{7}$ years ago, and worked for at least $10^{7}$ years.

We consider that cosmic rays at sufficiently large times of filling of the Galaxy spread uniformly over the Galactic plane. This follows from the observations. However, the diffusion model of propagation of cosmic rays in the Galaxy and the halo can not provide such uniform distribution with any distribution of sources $Q(\rho)$ over $\rho$ in the Galactic plane, where $\rho$ is the cylindrical radius. This is due to the fact that the second derivative $\partial^{2} N / \partial z^{2} \simeq N / H^{2}$ is not equal to zero. The value of $H$ is the vertical scale of the halo. Thus, in the steady 
state $\partial N / \partial \rho \neq 0$. Even the uniform distribution of sources in the Galactic plane $Q(\rho)=$ const,$\rho<R$, leads to the dependence $N(\rho)=\left(Q / \pi^{2} D R\right) E(\rho / R)$, where $E(x)$ is the complete elliptic integral of the second kind, which varies from $\pi / 2$ at $x=0$ to 1 at $x=1$ and has singularities of derivatives at $x=1$. For one source located in the center of the Galaxy, the distribution of $N(\rho)$ is also not uniform, $N(\rho) \propto 1 / \rho$. One way to overcome this contradiction is the assumption that the convective motion of cosmic rays, not only the diffusion motion, is very important in the Galaxy (Istomin [18]). The motion along the spiral arms, where there is a regular magnetic field, can occur with a speed close to the speed of light. Then in the steady state $\mathbf{v} \nabla N \simeq 0,|v| \simeq c$. If the source is located in the center of the Galaxy, the time to fill the Galaxy by cosmic rays is determined by two processes: the fast filling of arms during the time $\tau \simeq R / c \simeq 5 \cdot 10^{4}$ years; and slow diffusive overcoming of the central region of the Galaxy of the scale $L \simeq(1-2) \mathrm{kpc}$, and also of the interarm space of the same size approximately, $\tau \simeq L^{2} / D \simeq$ $10^{7}$ years. The total time to fill the Galaxy is determined by the slower process. However, the evacuation of cosmic rays from the Galaxy, if the source of cosmic rays stops working, differs from the filling, because the escape of particles is determined by the output into the outer regions of the Galaxy and the halo of the size of $R$, and has mainly a diffusive character. The time of the evacuation is of $\tau \simeq$ $R^{2} / D \simeq 10^{9}$ years. The given estimates refer to particles of small energy $E \simeq 1 \mathrm{GeV}$. For energetic particles the times of filling and escape are less due to an increase of the diffusion coefficient with increasing energy.

After the source is turned off particles continue to flow out from the Galaxy, and their density begins to decrease with time. Consider how this may happen. Suppose that at time $t=0$ cosmic rays with density $N_{0}(E)$ uniformly filled the spherical region (Galaxy and halo) of radius $R$. Then, assuming that the motion of particles is of diffusion character, using the Green function (equation (10)), we find the density of cosmic rays $N(r, E, t)$ at a point located at distance $r$ from the center of the Galaxy and at time $t$, $N(r, E, t)=N_{0}(E) F(p, r)$,

$$
\begin{aligned}
F(p, r)= & \frac{1}{\pi^{1 / 2}}\left\{\frac { R } { 2 r p } \left[\exp \left[-p^{2}(1-r / R)^{2}\right]\right.\right. \\
& \left.-\exp \left[-p^{2}(1+r / R)^{2}\right]\right] \\
& \left.+\int_{-p(1-r / R)}^{p(1+r / R)} \exp \left(-y^{2}\right) d y\right\}
\end{aligned}
$$

where the parameter $p$ is equal to $p(E, t)=\left[R^{2} / 4 D_{g}\right.$ $(E) t]^{1 / 2}$. For small values of $p, p<<1$, the function $F$ is equal to $F=4 p / \pi^{1 / 2}$, for large values $p>>1$, $F=1$. The transition of density of cosmic rays from the initial distribution $N_{0}(p>1)$ to the decrease with time, $N=N_{0}\left(4 R^{2} / \pi D_{g} t\right)^{1 / 2}$, takes place at $p \simeq 1$. At this point there is a transition of the spectrum of cosmic rays from the original, $N \propto E^{-2.7}$, with energies $E<E_{k}$ to the distribution of $N \propto E^{-2.7-0.7 / 2}=E^{-3.05}$ for $E>E_{k}$. The value of energy $E_{k}$ is defined by the equality $p=$ $1, D_{g}\left(E_{k}\right)=R^{2} / 4 t$. In the center of the Galaxy $(r=0)$ the transition occurs exactly at the point $p=1$, for us $(r \simeq R / 2)$, this point is also very close to unity. When $p=1$, there is a local increase of density of cosmic rays,

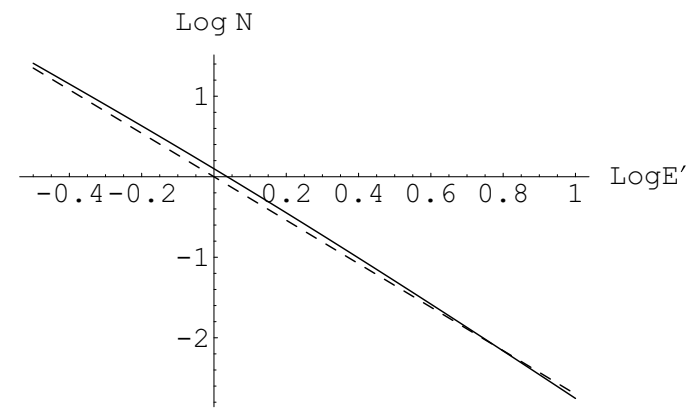

Figure 3. The distribution of Galactic cosmic rays $N(E) \propto$ $E^{-2.7} F(p)$ over energy $E$ near the "knee" at the point $r=R / 2, E^{\prime}=E / E_{k}$. The function $F(p)$ is defined by the expression (20). The dashed line is the dependence $N(E) \propto$ $E^{-2.7}$.

i.e. near the energy $E \simeq E_{k}$ the distribution $N(E)$ is really observed as the 'knee' (see Fig. 3).

It should be noted that results obtained do not depend on our assumption of a spherically symmetric distribution of Galactic cosmic rays in the disk and the halo. The distribution may have different scales in the plane of the Galaxy $R$ and across $H$. In diffusion models different scales $R$ and $H$ are due to the different diffusion coefficients of cosmic rays in the galactic plane, $D_{g}$, and in the direction above the Galactic plane, $D_{z}$. By that $D_{g} / R^{2}=D_{z} / H^{2}$. If we introduce a new coordinate above the Galactic plane $z^{\prime}=z\left(D_{g} / D_{z}\right)^{1 / 2}$, then in the coordinates $\left(r, z^{\prime}\right)$ the distribution of rays will be spherically symmetric. Because we are interested in the distribution in the Galactic plane, $z=0$, it will be the same as at $z^{\prime}=0$.

The position of the 'knee' depends on the time $t$, passed after turning off the source. Knowing the position of the 'knee' at the present time $t=t_{0}$, we find

$$
\begin{aligned}
t_{0} & =\frac{R^{2}}{4 D_{g}\left(E_{k}\right)}=4.2 \cdot 10^{4}\left(\frac{R}{5 \cdot 10^{22} \mathrm{~cm}}\right)^{2} \\
& \times\left(\frac{D_{g}(1 \mathrm{GeV})}{2.2 \cdot 10^{28} \mathrm{~cm}^{2} \mathrm{~s}^{-1}}\right)^{-1}\left(\frac{E_{k}}{3 \cdot 10^{15} \mathrm{eV}}\right)^{-0.7} \text { year. }
\end{aligned}
$$

It should be noted that the 'knee' is moving with time, $E_{k}=3 \cdot 10^{15}\left(t / t_{0}\right)^{-1.43} \mathrm{eV}$. The speed is now equal to $d E_{k} / d t=-1.43 E_{k} / t_{0}=-10^{11} \mathrm{eV} /$ year.

\section{Conclusions}

We showed that the distribution of power of sources of extragalactic cosmic rays in the energy range of 3 . $10^{18} \mathrm{eV}<E<10^{21} \mathrm{eV}$ is a power law, $\bar{q}(E) \propto E^{-2.7}$. Thus, it is the same as in our Galaxy in the energy range below the 'knee', $E<3 \cdot 10^{15} \mathrm{eV}$. This indicates the common nature of the origin of cosmic rays in the Galaxy and in other galaxies. However, the power of extragalactic sources exceeds by at least two orders of magnitude the capacity of the Galaxy (see formula (19)). The conclusion from this is that a 'normal' galaxy, to which our Galaxy belongs, is not the source of cosmic rays. The estimation of the density of extragalactic cosmic rays sources, $N_{g} \simeq$ $10^{-2}-10^{-3} \mathrm{Mpc}^{-3}$, indicates galaxies with active nuclei. The possible source of energetic charged particles is 
relativistic jets emitted from the surroundings of massive black holes. Thus, acceleration by shock waves from supernova explosions is not possible to explain the origin of cosmic rays up to energies $\simeq 10^{18} \mathrm{eV}$. Otherwise, all galaxies in more or less degree would be sources of cosmic rays, because in all of them there are supernova explosions. Why, nevertheless, we observe cosmic rays in the Galaxy is explained that once in the past the Galaxy was also active. From the center of the Galaxy there emitted a relativistic bipolar jet, the remnants of which is observed now above and below the Galactic plane as bubbles of relativistic gas with the power law energy spectrum $E^{-2}(\mathrm{Su}$, Slatyer \& Finkbeiner [21]).

Due to the fact that this source of cosmic rays in the Galaxy is now not working, it appears the deflection of the spectrum of Galactic cosmic rays from the dependence $N(E) \propto E^{-2.7}$ in the whole energy range. For $E>3$. $10^{15} \mathrm{eV}$, the spectrum becomes softer, $N(E) \propto E^{-3.1}$. This is because particles, that once filled the Galaxy, leave it faster the larger their energy, because their diffusion coefficient increases with energy, $D \propto E^{0.7}$. Particles of larger energies leave the Galaxy quicker than particles of lesser energies. For the diffusive motion of particles, $N(E) \propto N_{0}(E)(D t)^{-1 / 2} \propto E^{-2.7-0.7 / 2}$ for energies $E>$ $E_{k}$ and $N(E) \simeq N_{0}(E)$ for energies $E<E_{k}$, where $N_{0}(E)$ is the initial distribution of particles. Thus, the 'knee' formation reflects the escape of particles from the Galaxy. Knowing the current position of the 'knee' one can estimate the time when the source of cosmic rays in the Galaxy stopped, it occurred $4.2 \cdot 10^{4}$ years ago. The position of the 'knee' is not constant in time, it must move, $E_{k} \propto t^{-1.43}$. This motion can be noticed if we have a sufficient accuracy of the measurement of the 'knee' position. During the last 50 years the 'knee' has moved by $\Delta E_{k}=-5 \cdot 10^{12} \mathrm{eV}$.

The release of cosmic rays from the Galaxy also explains the appearance of heavy nuclei in the energy range $10^{16} \mathrm{eV}<E<10^{18} \mathrm{eV}$, just between the knee and extragalactic particles. Indeed, the particle Larmor radius is inversely proportional to the nucleus charge $Z$. For a fixed energy particle diffusion is less for heavy nuclei than for protons. Protons leave the Galaxy faster.

The recent discovery of the source of $\mathrm{PeV}$ particles in the Galactic center with the power of $10^{37-38} \mathrm{erg} / \mathrm{s}$, made by the HESS group [22], confirms the hypothesis that centers of galaxies with massive black holes are powerful sources of ultrahigh energy particles. If we take into account the fact that the luminosity of Sgr A* is now
$10^{-9}$ of the Eddington luminosity, one can imagine what will be the luminosity of the Galactic center in cosmic rays during its active phase.

Author thanks V. Berezinsky for fruitful discussions. This work was done under support of the Russian Foundation for Fundamental Research (grant numbers 14-02-00831 and 15-0203063).

\section{References}

[1] Krymskii, G.F., Soviet Physics-Doklady 22, 327 (1977)

[2] Bell, A.R., MNRAS 182, 147 (1978)

[3] Blasi, P., The Astronomy and Astropart. Review 21, 70 (2013)

[4] Pierre Auger Collaboration, 2010, Astropart. Phys. 34, 314 (2010)

[5] Pierre Auger Collaboration, Science 318, 939 (2007)

[6] Pierre Auger Collaboration, Astropart. Phys. 29, 188 (2008)

[7] Greisen, K., Phys. Rev. Lett. 16, 748 (1966)

[8] Zatsepin, G.T., Kuz'min, V.A., JETP Letters 4, 78 (1966)

[9] Stanev, T., Engle, R., Mucke A., Protheroe, R.J., Rachen, J.P., Phys. Rev. D 62, 093005 (2000)

[10] Berezinsky, V., Gazizov, A., Grigorieva S., Phys. Rev. D 74, 043005 (2006)

[11] Aloisio, R., Berezinsky V., Grigorieva, S., Astropart. Phys. 41, 94 (2013)

[12] Takami, H., Sato, K., Astropart. Phys. 30, 306 (2009)

[13] Berezinsky, V., EPJ Web of Conference 53, 01003 (2013)

[14] Tinyakov, P. for the Telescope Array Collaboration, Nuclear Instruments and Methods in Physics Reseach A 724, 29 (2014)

[15] Taylor, A.M., Ahlers, M., Aharonian F.A., Phys. Rev. D 84, 10500 (2011); ArXiv: astro-ph/1107. 2055

[16] Mao-Li, M., Xin-Wu, C., Dong-Rong, J., Min-Feng, G., Chin. J., Astron. Astrophys. 8, 39 (2008)

[17] Abbasi, R.U., et al., ApJ 610, L73 (2004)

[18] Istomin, Ya.N., New Astronomy 27, 13 (2014)

[19] Ptuskin, V.S., Phys. Uspechi 50, 534 (2007)

[20] Blasi, P., Amato, E., JCaP Issue 1, id. 011 (2012)

[21] Su, M., Slatyer, T.R., Finkbeiner, D.P., ApJ 724, 1044 (2010)

[22] HESS Collaboration, Nature 531, Issue 7595, 476 (2016) 